\title{
Activity-Dependent Regulation of Mitochondrial Motility by Calcium and Na/K-ATPase at Nodes of Ranvier of Myelinated Nerves
}

\author{
Chuan Li Zhang, ${ }^{1}$ Po Lai Ho, ${ }^{1}$ Douglas B. Kintner, ${ }^{2}$ Dandan Sun, ${ }^{2}$ and Shing Yan Chiu ${ }^{1}$ \\ Departments of ${ }^{1}$ Physiology and ${ }^{2}$ Neurological Surgery, University of Wisconsin School of Medicine and Public Health, Madison, Wisconsin 53706
}

The node of Ranvier is a tiny segment of a myelinated fiber with various types of specializations adapted for generation of high-speed nerve impulses. It is ionically specialized with respect to ion channel segregation and ionic fluxes, and metabolically specialized in ionic pump expression and mitochondrial density augmentation. This report examines the interplay of three important parameters (calcium fluxes, Na pumps, mitochondrial motility) at nodes of Ranvier in frog during normal nerve activity. First, we used calcium dyes to resolve a highly localized elevation in axonal calcium at a node of Ranvier during action potentials, and showed that this calcium elevation retards mitochondrial motility during nerve impulses. Second, we found, surprisingly, that physiologic activation of the Na pumps retards mitochondrial motility. Blocking Na pumps alone greatly prevents action potentials from retarding mitochondrial motility, which reveals that mitochondrial motility is coupled to Na/K-ATPase. In conclusion, we suggest that during normal nerve activity, Ca elevation and activation of $\mathrm{Na} / \mathrm{K}$-ATPase act, possibly in a synergistic manner, to recruit mitochondria to a node of Ranvier to match metabolic needs.

\section{Introduction}

Mitochondria (Andrews et al., 2005, 2006; Dutta et al., 2006; Einheber et al., 2006; Baloh et al., 2007; Mahad et al., 2008, 2009), $\mathrm{Ca}^{2+}$ (Sun and Chiu, 1999; Verbny et al., 2002; Zhang et al., 2004, 2006; Stys, 2005), and Na/K-ATPase (Waxman, 2008; Young et al., 2008) are three important players in normal physiology and pathophysiology of myelinated axons. In particular, dysregulation of mitochondrial dynamics, such as motility, is linked to axonal pathology (Baloh et al., 2007; Chan, 2007; Detmer and Chan, 2007; Baloh, 2008). This study addresses a novel question in myelinated nerve biology on this interesting tripartite: mitochondrial motility, $\mathrm{Ca}$, and $\mathrm{Na}$ pumps. How does nerve activity affect interaction within this tripartite?

Mitochondria are not uniformly distributed in neurons (Hollenbeck and Saxton, 2005) and display increased density at two major, metabolically active sites: the nodes of Ranvier in the peripheral nervous system (Berthold, 1978; Fabricius et al., 1993) and the synapse (for review, see Hollenbeck and Saxton, 2005). A local increase in mitochondrial density may reflect rapid recruitment of these highly mobile organelles to active sites to serve metabolic demands during physiological stimuli. Of these two sites, mitochondrial recruitment has been thoroughly studied in the synapse. In the synapse, the primary signal for recruitment is high Ca influx mediated by neurotransmitters (Yi et al., 2004), which arrests mitochondria through a direct and indirect mech-

\footnotetext{
Received Sept. 14, 2009; revised Nov. 13, 2009; accepted Dec. 24, 2009

This work was supported in part by National Multiple Sclerosis Society Grant RG4054-A-8 (S.Y.C.), National Institutes of Health Grant R01NS48216 (D.S.), and American Heart Association Grant EIA 0540154 (D.S.).

Correspondence should be addressed to Dr. Shing Yan Chiu at the above address. E-mail: chiu@physiology. wisc.edu.

DOI:10.1523/JNEUROSCI.4551-09.2010

Copyright $\odot 2010$ the authors $\quad 0270-6474 / 10 / 303555-12 \$ 15.00 / 0$
}

anism. Directly, calcium arrests mitochondrial motility by targeting Miro, a calcium sensor coupled to mitochondrial motors (Saotome et al., 2008; Cai and Sheng, 2009; Macaskill et al., 2009; Wang and Schwarz, 2009). Indirectly, high Ca influx arrests mitochondrial movement by local ATP depletion caused by activation of Ca-ATPase [i.e., sarco-endoplasmic reticulum $\mathrm{Ca}^{2+}$-ATPase (SERCA) on the endoplasmic reticulum (ER)] (Brough et al., 2005; Mironov, 2006, 2007).

In contrast, nothing is known about regulation of mitochondrial motility at nodes of Ranvier during excitation. This issue is of particular interest for two reasons. First, the marked difference in excitation mechanism between a synapse $\left(\mathrm{Ca}^{2+}\right.$ influx mediated by neurotransmitters) and a node ( $\mathrm{Na}$ influx mediated by voltage) suggests that in a node, Na rather than Ca might be the key signal for regulation of mitochondrial motility. A node of Ranvier is uniquely adapted for $\mathrm{Na}$ signaling: it has a very high $\mathrm{Na}$ channel density (Chiu, 1980) and a very small cytosolic volume that produces robust intracellular $\mathrm{Na}$ signaling that in principle can be coupled to mitochondrial motility. Could this coupling be mediated by Na-pump activation and local ATP depletion? Second, direct Ca-mediated stoppage of mitochondria is possible at a node of Ranvier. Recent studies have demonstrated axonal calcium signaling mediated by neurotransmitter (Sun and Chiu, 1999; Zhang et al., 2004) and voltage (Zhang et al., 2006). However, no study has yet definitively resolved Ca signaling at a node of Ranvier.

This study reports two important findings. First, we successfully resolved calcium signaling at a node of Ranvier during action potentials. Second, we discovered a novel form of signaling for the $\mathrm{Ca} / \mathrm{Na}$-pump/mitochondria tripartite: during nerve impulses, Ca elevation and Na-pump activation retard mitochondrial motility at a node, possibly in a highly synergistic manner. 


\section{Materials and Methods}

Animals. Sciatic nerves from male adult frogs (Xenopus laevis) were used. Experiments were performed at room temperature.

Imaging of axonal calcium. Sciatic nerves were dissected from frogs, desheathed, and the axons selectively loaded with a membraneimpermeant calcium dye following a published work from our laboratory (Zhang et al., 2006). We used the cell-impermeant, high-affinity Oregon Green 488 BAPTA-1 [OGB-1; $K_{\mathrm{d}}=170 \mathrm{~nm}$; molecular weight (MW), 1114] conjugated to the high molecular weight Dextran (MW, 10,000 ; Invitrogen). Briefly, the dye was introduced into the axonal cylinders by diffusion and/or axonal transport using a tight suction pipette (containing 1-5 $\mathrm{mm}$ of dye in $120 \mathrm{~mm} \mathrm{KCl}$ titrated to $\mathrm{pH} 7.3$ ) applied to one cut end of the nerve. The other end was used for stimulation with bipolar electrodes. Experiments typically began after 3-4 h of dye loading. We typically performed calcium imaging at a site at least $1 \mathrm{~cm}$ away from the dye-loading pipette. The dyes were allowed to remain in the loading pipette (which also served as the recording pipette) during the entire experiment. Calcium levels were not reported in absolute levels but as $\Delta F / F_{0}$, where $F_{0}$ is the baseline fluorescence signal.

Calcium images were viewed with a $60 \times$ (Olympus) objective lens coupled to a Yogokawa spinning disc system. OGB-1 was used at a concentration of 1-5 $\mathrm{mm}$ in the loading pipette; the actual axonal dye concentration at the optical recording site was estimated previously (Verbny et al., 2002) to be $\sim 1.3 \%$ of the dye concentration at the loading pipette, which would have an upper range of $\sim 65 \mu \mathrm{M}$. The dyes were excited by an argon laser at $488 \mathrm{~nm}$, and confocal fluorescence signals were collected through a $500 \mathrm{~nm}$ long-pass emission filter. Images of myelinated axons with visible nodes of Ranvier were selected, and calcium images were streamed at a rate of $40 \mathrm{~ms}$ between frames. Image acquisition and online calculations were controlled through MetaMorph software (Universal Imaging).

Imaging of mitochondrial motility. Frogs were anesthetized by submersion in water containing ethyl 3-aminobenzoate methanesulfonate (20 $\mathrm{g} / \mathrm{L}$ water) for $10-15 \mathrm{~min}$. The skin was cut, and the sciatic nerve was exposed near the sciatic notch (the site where the nerve exits the spinal cord). A sharp glass micropipette, mounted to a holder and controlled by a fine three-dimensional manipulator, was used to locally inject MitoTracker Green into the sciatic nerve. The injection pipette contained 0.5 mM MitoTracker dissolved in dimethyl sulfoxide, and $\sim 2 \mu$ l was gently injected under visual guidance through a microscope at $20 \times$ magnification. The frog was allowed to recover from surgery. At $24 \mathrm{~h}$ after surgery, the frog was killed, and the sciatic nerves were taken out for experiments. During the $24 \mathrm{~h}$, the locally injected bolus of MitoTracker was taken up by Schwann cells and axons at the injection site. However, only the axonal mitochondria and not the Schwann cell mitochondria labeled at the site of injection were transported away from the injection site. As a result, at $24 \mathrm{~h}$ after injection, a nerve segment $\sim 2-3 \mathrm{~cm}$ downstream from the injection site contained pure axonal MitoTracker-labeled mitochondria. The sciatic nerves (total length of about $5-6 \mathrm{~cm}$ ) were gently dissected and mounted on a nerve chamber for image analysis of mitochondrial motility. The labeled mitochondria are visualized with the same optics for visualizing calcium dye (i.e., excited at $488 \mathrm{~nm}$ and MitoTracker Green confocal fluorescence signals collected through a $500 \mathrm{~nm}$ longpass emission filter). Each image was integrated for $500 \mathrm{~ms}$, and movies of mitochondrial motility were captured one frame every $2 \mathrm{~s}$ for a duration of 1-2 min. If necessary, nerves were stimulated at one end with bipolar electrode to generate compound action potentials that were measured at the other end with a suction pipette.

After an experiment, the images were subjected to motility analysis by generating kymographs using Image (NIH). From these kymographs, several motility parameters [number of mobile mitochondria $(N)$, velocity $(V)$, and pause duration $(P)]$ were extracted for quantitative analysis. These parameters and their extraction criteria are described in Results.

We are aware that there are transgenic mice with genetically labeled mitochondria that would facilitate mitochondrial motility studies. However, we have no access to these mice. On the other hand, there are good reasons for selecting frog (Xenopus) for these studies. First, frog sciatic nerves are robust and allow long-term measurement of stable mitochon- drial motility. Second, historically, the pioneering works on mitochondrial motility in myelinated axons were all done in frog (Cooper and Smith, 1974). Our work extends these earlier studies and provides major new information about mitochondrial dynamics.

Electrophysiology. In both calcium-imaging and mitochondrial motility studies, compound action potentials were evoked by a $125 \%$ supramaximal stimulus applied via a bipolar electrode to one cut end, and recorded at the other cut end through another suction electrode. The amplitude of the compound action potential (CAP) data was analyzed using pClamp 6.0 software (Molecular Devices).

Solutions and drugs. Sciatic nerves were normally bathed in a frog Ringer's solution containing the following (in $\mathrm{mm}$ ): $100 \mathrm{NaCl}, 3 \mathrm{KCl}, 2$ $\mathrm{CaCl}_{2}, 1 \mathrm{MgSO}_{4}, 3$ HEPES, $20 \mathrm{NaHCO}_{3}, 10$ glucose, 2.5 sodium pyruvate, and 2.5 malic acid. All solutions were rigorously oxygenated with $95 \% \mathrm{O}_{2}$ and $5 \% \mathrm{CO}_{2}$. Calcium-free solutions were prepared by replacing $\mathrm{Ca}^{2+}$ with $\mathrm{Mg}^{2+}$ to keep the external divalent cation concentration constant, and by adding EGTA ( $1 \mathrm{~mm}$ ); $\mathrm{pH}$ was adjusted to 7.4 with $\mathrm{NaOH}$ or $\mathrm{HCl}$ as necessary. For the $\mathrm{Na}$-free solution, $\mathrm{NaCl}$ was replaced by $\mathrm{LiCl}$ (100 mM), and $\mathrm{NaHCO}_{3}$ was replaced by choline bicarbonate $(20 \mathrm{~mm})$. OGB-1 and MitoTracker Green were purchased from Invitrogen. All other chemicals were from Sigma. All dye-loading and calcium-imaging experiments were done at room temperature $\left(20-22^{\circ} \mathrm{C}\right)$.

\section{Results}

The Results section has two parts. In part one, we describe how we resolved calcium signaling in single nodes of Ranvier and briefly examine the mechanism for calcium signaling. In part two, we focus on mitochondrial motility and examine how nodal $\mathrm{Ca}$ and other factors regulate mitochondrial movement during nerve impulses.

\section{Action potentials evoked localized axonal Ca rise at nodes of Ranvier}

Before examining roles of calcium in mitochondrial motility, we need to first examine whether there is activity-dependent elevation of axonal calcium at a node of Ranvier. The issue of $\mathrm{Ca}$ signaling at a node has not been settled, despite recent advances in imaging axonal $\mathrm{Ca}$ in myelinated nerves (Sun and Chiu, 1999; Verbny et al., 2002; Zhang et al., 2006). We have now successfully addressed this issue in frog nodes. Figure $1 A$ (left) shows nodal calcium elevation triggered by a brief train of action potentials ( $4 \mathrm{~s}$ at $25 \mathrm{~Hz}$ ). Time-lapsed fluorescence images were collected from a myelinated single axon in an unteased sciatic nerve bundle. Cytosolic calcium was reported by introducing cellimpermeant, Dextran-conjugated Oregon Green BAPTA-1 selectively into axon cylinders through cut ends. Many axons were labeled, from which only one was selected for study. Single node of Ranvier was easily identified through light microscopy (Fig. $1 A$, top). The key observation is that the evoked axonal calcium elevation was highly localized to the node, spreading only by $\sim 20$ $\mu \mathrm{m}$ into internodes of $\sim 2000 \mu \mathrm{m}$ in an adult frog. To see if the $\mathrm{Ca}$ spread into the internodes could be augmented by stronger stimulation, we either increased the frequency of stimulation (Fig. $1 B-D$ ) or prolonged the duration of stimulation from 1 to $2 \mathrm{~min}$ (Fig. $1 E$ ). Both procedures failed to enlarge the spatial spread of the nodal Ca response, suggesting a strong buffering mechanism that prevents the spread of calcium into the internodes. This localized nodal response in the PNS myelinated axons stands in striking contrast to CNS myelinated axons where the evoked Ca response is spatially uniform (Zhang et al., 2006). We next examined the mechanisms underlying the nodal calcium response.

\section{Calcium-free bath solutions and calcium channel blockers}

To examine whether calcium influx underlies the evoked calcium transient, we eliminated extracellular calcium from the bath solu- 

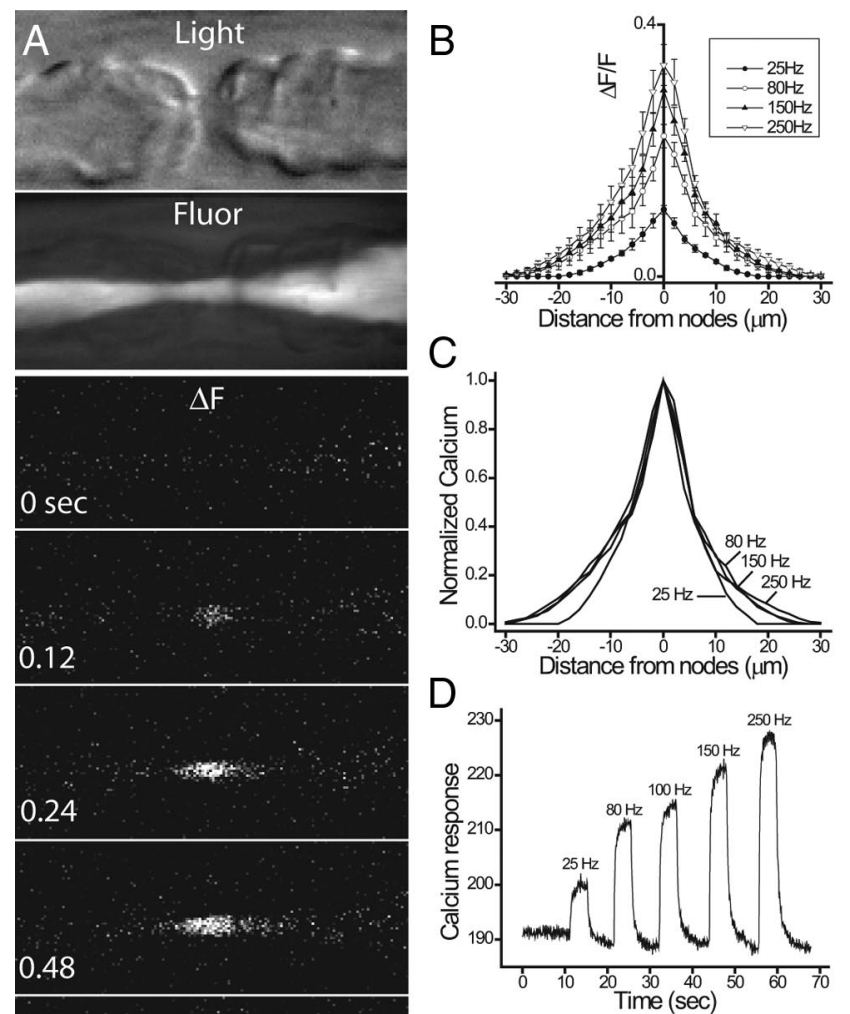

0.48
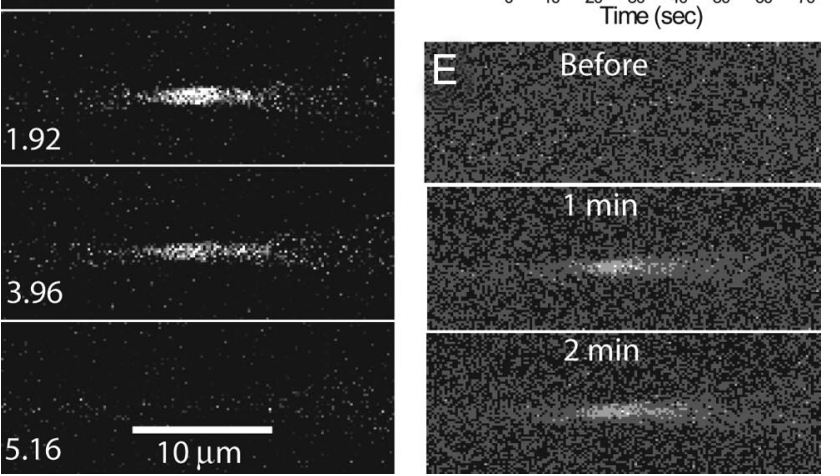

Figure 1. Action potentials trigger localized Ca rise at nodes of Ranvier in frog myelinated axons. $\boldsymbol{A}$, Time-lapsed Ca fluorescent images showing nodal Ca response evoked by repetitive action potentials ( $4 \mathrm{~s}$ at $25 \mathrm{~Hz}$ ). The top two images are light microscopic and fluorescent images, respectively, of a single node with adjacent paranodes. The fluorescent image shows that only the axon cylinder is labeled with the cell-impermeant calcium dye 0GB-1. The rest of the images show fluorescent-subtracted images $(\Delta F)$ from the control (time, $0 \mathrm{~s}$ ) at various times during and after stimulation. The stimulation was turned off at $4 \mathrm{~s}$. The evoked Ca response centers at the node and spreads to no more than $\pm 20 \mu$ m into the two paranodes. $B, C$, Spatial distribution of evoked Ca response. The node is positioned at 0 . The frequency of stimulation was increased from 25 to $250 \mathrm{~Hz}$ at a fixed duration of $4 \mathrm{~s}$. The amplitude of evoked $\mathrm{Ca}$ response increased with stimulation frequency $(\boldsymbol{B})$, but the spatial spread into the paranodes $(\boldsymbol{C}$; normalized with respect to the peak) remained unaffected $(N=5)$. $\boldsymbol{D}$, Temporal response of nodal Ca signal evoked by increasing stimulation frequency. $E$, Nodal Ca responses remain localized even at prolonged stimulation. Fluorescent-subtracted images (from control) at 1 and 2 min of continuous stimulation at $200 \mathrm{~Hz}$.

tions ( 2 mm Ca replaced by Mg, with 1 mm EGTA added). Figure $2 \mathrm{~A}$ shows that $15 \mathrm{~min}$ of $\mathrm{Ca}$-free bath solution reversibly blocked the evoked calcium response without affecting the single CAP (shown on top with a different time scale). In some experiments (as in this one), there was a fall in the resting Ca fluorescence, suggesting a fall in resting cytosolic calcium during Ca-free solution application.

To examine possible contribution of voltage-gated calcium channels, we applied two wide spectrum blockers of voltage-gated calcium channels, $\mathrm{La}^{3+}(100 \mu \mathrm{M}$; one of the most potent blockers
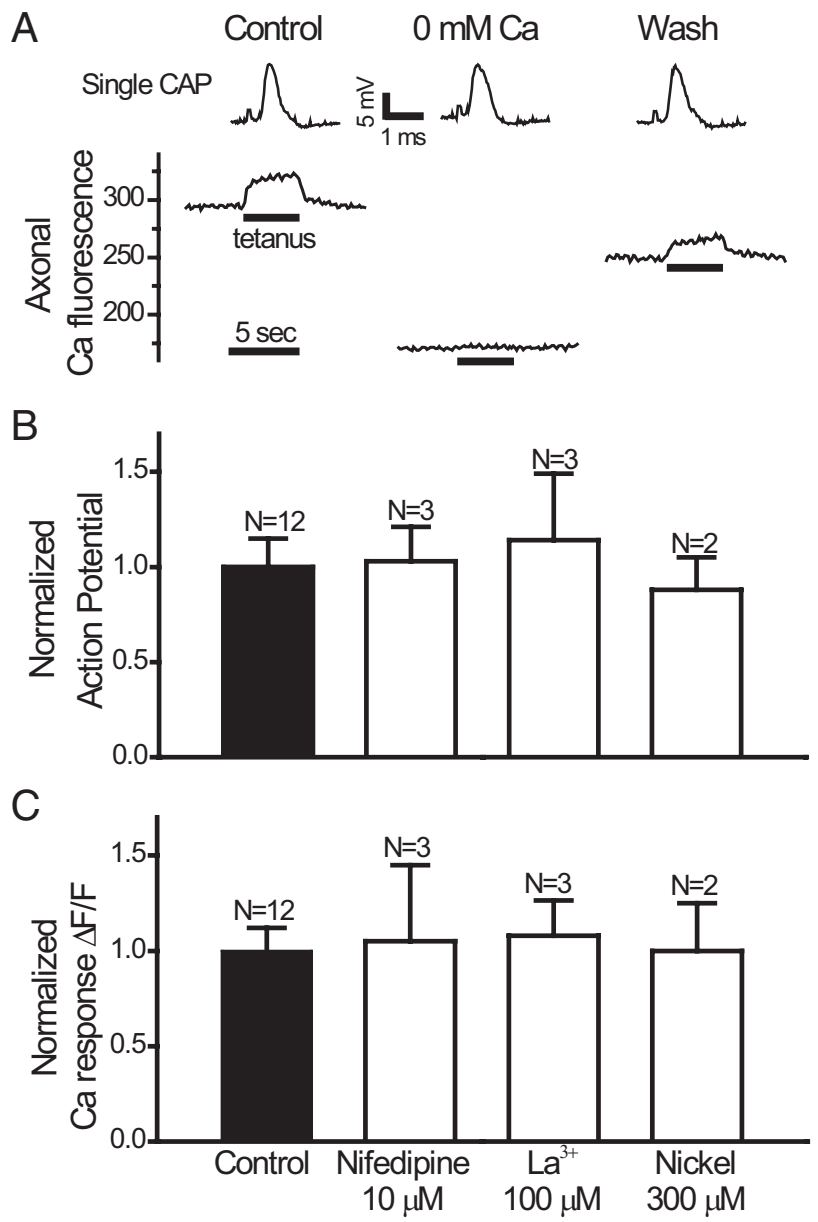

Figure 2. Effects of calcium-free solutions and $\mathrm{Ca}$ channel blockers on the evoked nodal $\mathrm{Ca}$ response. $A$, Top, Single CAPs in response to Ca-free solutions. Bottom, Axonal Ca fluorescence during a tetanus $(25 \mathrm{~Hz}, 4 \mathrm{~s})$ before and after $\mathrm{Ca}$-free bath solutions. Note the difference in time scale between top and bottom. $\boldsymbol{B}$, Normalized compound action potential amplitude in the presence of various Ca channel blockers. C, Corresponding evoked Ca responses in the presence of various Ca channel blockers. Error bars indicate SEM.

with $K_{\mathrm{d}}$ near the 1-2 $\mu \mathrm{M}$ range) and $\mathrm{Ni}^{2+}(300 \mu \mathrm{M})$. Neither the compound action potentials (Fig. $2 \mathrm{~B}$ ) nor the evoked Ca response (Fig. 2C) were affected by these two blockers. The L-type calcium channel blocker nifedipine $(10 \mu \mathrm{M})$ was also without effects on the evoked Ca response (Fig. 2C). The calcium-free studies suggest that evoked Ca response was mediated by calcium influx, but the negative results of the wide-spectrum Ca channel blockers suggest that novel influx pathway may be involved.

\section{Mitochondrial blockers}

To examine possible contribution of mitochondria to the evoked calcium response, we applied various agents to block mitochondrial functions. First, we blocked mitochondrial function with protonophore carbonyl cyanide $p$-trifluoromethoxyphenylhydrazone (FCCP), which dissipates the proton gradient and should lead to emptying of mitochondrial calcium. Figure $3 A$ shows that FCCP (15 min application) inhibits the evoked calcium response without affecting the compound action potentials. The inhibition of the calcium response is not the result of blocking ATP production, since selectively blocking mitochondrial ATPase with oligomycin did not block the calcium response (Fig. 3B). Furthermore, the inhibition of the calcium response by FCCP was unaffected when oligomycin was coapplied (Fig. 3B). 
Second, we bath applied 7-chloro-5-(2chlorophenyl)-1,5-dihydro-4,1-benzothiazepin-2(3H)-one [CGP-37157 (CGP)] to block calcium release from the mitochondrial $\mathrm{Na} / \mathrm{Ca}$ exchanger (Cox et al., 1993; Baron and Thayer, 1997; David, 1999; Yang et al., 2003). CGP (10-50 $\mu \mathrm{M})$ blocks the evoked calcium response without affecting the compound action potentials (Fig. 3C,D), and the CGP effect was partially reversible after extended wash (Fig. 3C). CGP is known to block L-type calcium channels. However, nifedipine is without effect on the evoked Ca response (Fig. 2C). Hence, CGP appears to block nodal Ca response by blocking mitochondrial calcium release. Collectively, the effects of FCCP (which empties mitochondria of calcium) and CGP (blocking mitochondrial $\mathrm{Ca}$ release) suggest that mitochondrial calcium release contributes to the evoked calcium response. Although the calcium-free studies (Fig. 2A) suggest an influx component, it is possible that the results of the calcium-free studies reflect a rapid depletion of mitochondrial calcium by calcium-free bath solutions (Pitter et al., 2002).

\section{ER blockers}

To assess the role of ER, we emptied ER calcium by blocking the calcium pump (SERCA) on the ER with $2 \mu \mathrm{M}$ thapsigargin (Thastrup et al., 1990). Thapsigargin treatment (15 min) blocked the nodal calcium response without affecting the compound action potentials (data not shown). This suggests a direct contribution of the ER to the calcium response. Alternatively, thapsigargin may have indirect effects on mitochondrial function resulting from prolonged ER blockage (Hom et al., 2007).

To summarize the first part of this paper, using a method of selective labeling of axons with $\mathrm{Ca}$ dyes, we have decisively localized activity-dependent $\mathrm{Ca}$ signaling to the node of Ranvier in frog myelinated axons. The mechanism for the calcium response appears complex, and likely involves contributions from both influx and release from intracellular organelles such as mitochondria.

We now move to the second major part of this study in which we will examine the role of evoked Ca response and other factors in regulating nodal mitochondrial motility during action potentials. We launched a new series of studies in which calcium dyes were not involved, but in which we instead focused on monitoring mitochondrial motility with MitoTracker Green.

\section{Real-time analysis of mitochondrial motility in myelinated axons}

MitoTracker Green was injected locally into sciatic nerves near the sciatic notch of anesthetized frogs, and the nerves were taken
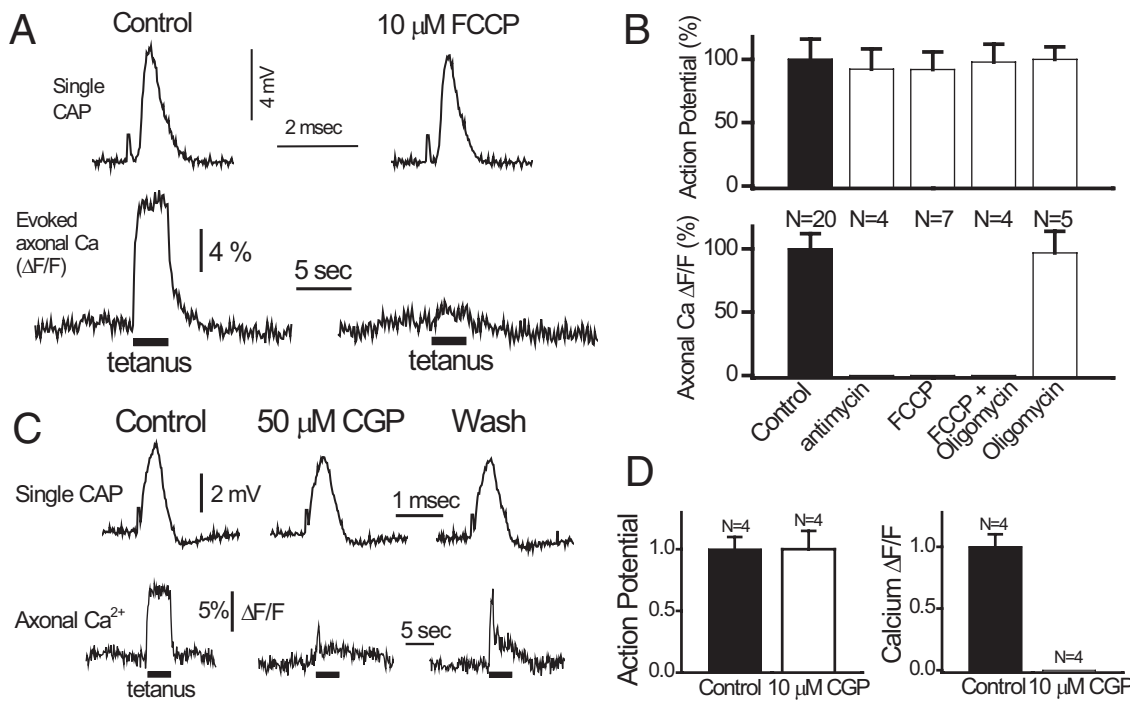

Figure 3. Effects of FCCP and CGP on compound action potential and evoked nodal Ca response. $A, \mathrm{FCCP}(10 \mu \mathrm{m}, 15 \mathrm{~min})$ blocks the evoked Ca response (bottom) but not the compound action potential (top). $\boldsymbol{B}$, Averaged results for various blockers of the electron chain complex (antimycin, $20 \mu \mathrm{m} ; \mathrm{FCCP}, 10 \mu \mathrm{m}$ ) and the mitochondrial ATPase (oligomycin, $10 \mu \mathrm{m}$ ). Note that all compounds tested ( $15 \mathrm{~min}$ ) did not affect the compound action potentials (top). C, CGP (50 $\mu \mathrm{m}, 30 \mathrm{~min}$ ) blocks the evoked nodal Ca response. The effect is reversible after extended wash $(50 \mathrm{~min})$. D, Averaged results for CGP at $10 \mu \mathrm{M}$. CGP blocks evoked Ca response (right) without affecting the compound action potential (left). Error bars indicate SEM.
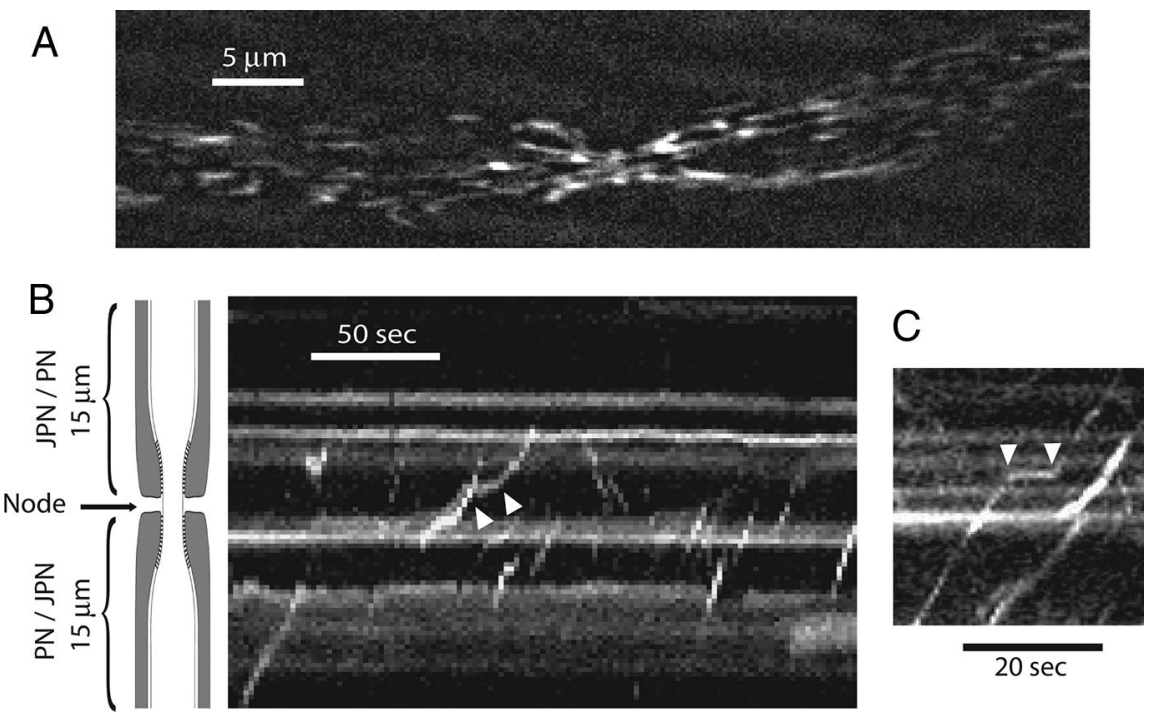

Figure 4. Kymographs of mitochondrial motility. $\boldsymbol{A}$, Still fluorescent image of MitoTracker-labeled mitochondria in the axon of a single myelinated fiber. The node is near the center where the axon shows a conspicuous constriction (supplemental Movie 1, available at www.jneurosci.org as supplemental material). $\boldsymbol{B}, \boldsymbol{C}$, Representative kymographs showing anterograde movement (positive slope), retrograde movement (negative slope), and pauses (between white arrowheads). The schematic drawing of myelinated axon applies to $\boldsymbol{B}$ and $\boldsymbol{C}$. Note the position of the node and the flanking paranodes (PN) and juxtaparanode (JPN).

for experiments $24 \mathrm{~h}$ after injection. The nerves were gently desheathed but remained unteased, and were cut to about 5-6 $\mathrm{cm}$ long. Studies of axonal mitochondrial motility were performed in the middle of a nerve bundle, about $2 \mathrm{~cm}$ downstream from the site of injection. Confocal image analysis was performed on single myelinated axons located near the surface of the nerve bundle. Figure $4 \mathrm{~A}$ shows a still frame of a MitoTracker-labeled myelinated axon from a movie used to visualize mitochondrial motility (supplemental Movie 1, available at www.jneurosci.org as supplemental material). The node is located near the middle where the axon is constricted. Axonal mitochondria can be seen 
moving in anterograde (the majority) and retrograde directions at a velocity of $0.2-2.0 \mu \mathrm{m} / \mathrm{s}$. Mitochondrial movements in single axons persist undiminished for at least $2 \mathrm{~h}$. We restricted our analysis to changes in mitochondrial motility induced by various experimental conditions over a brief period of 1-10 min. In all studies, only one node per nerve bundle was used for each experiment.

Motility analysis for mitochondria was performed by plotting kymographs using Image $(\mathrm{NIH})$. Motility analysis was restricted to an axonal length of $10 \mu \mathrm{m}$ with the node in the middle. Figure 4, $B$ and $C$, shows representative kymographs from a narrow slice parallel to the axis of the axon. Each slice is $0.69 \mu \mathrm{m}$ thick and is oriented longitudinally through the node. In our presentation of kymographs, the vertical axis is the distance along a slice, and the horizontal axis is the time. A schematic of a myelinated axon is shown on the left of Figure $4 B$ to show the location of the node. Movements of mitochondria along a longitudinal slice produce a trajectory consisting typically of a straight line with either a positive slope (anterograde movement) or negative slope (retrograde movement). Mitochondria rarely moved along the entire $10 \mu \mathrm{m}$ length of a kymograph slice (only $0.69 \mu \mathrm{m}$ thick) during the observation period. Frequently, mitochondria moved for a short distance along a particular kymograph slice, then were picked up again as they moved into an adjacent kymograph slice. Sometimes, mitochondrial movement (in both directions) shows momentary pauses, as indicated by the pair of white arrows (Fig. $4 B, C)$. Stationary mitochondria are represented by a horizontal line. From these kymographs, we extracted three major parameters for study: number of mobile mitochondria $(N)$, velocity of mobile mitochondria $(V)$, and pause duration $(P)$.

\section{Number of mobile mitochondria}

We determined the total number of mitochondria moving through a node during a fixed period (typically 1 or $2 \mathrm{~min}$ ). To obtain this number, we produced a series of adjacently spaced kymographs (fixed at 14 slices; i.e., a total of $9.66 \mu \mathrm{m}$ ) that covered the entire node. By comparing adjacent kymographs, we followed the movement of the same mitochondria as they moved through different slices and distinguished them from new mitochondria that moved into the view at later times. This number, $N$, remains the same as the same mitochondria are followed through adjacent kymographs, and $N$ is increased accordingly when adjacent kymographs revealed new mitochondria that moved into the viewing area. It is important to emphasize that we currently did not have enough resolution to resolve a single mitochondrion. Thus, each trajectory could either be a single mitochondrion or a single mitochondrial mass composed of several mitochondria moving together as a unit. Throughout this paper, we will refer to $N$ as the number of mobile mitochondria, with the understanding that it is actually a measure of the number of different, discrete mitochondrial masses that are mobile under different experimental conditions.

\section{Velocity of mobile mitochondria}

From the slope of the movement trajectory in the kymographs, we determined the velocity of each discrete mobile mitochondrial mass for both anterograde and retrograde directions. Velocity for a given mitochondrial mass was taken from a particular kymograph showing a long trajectory, ignoring minor changes than might have occurred for the same mitochondrial mass as it moved through other kymographs.
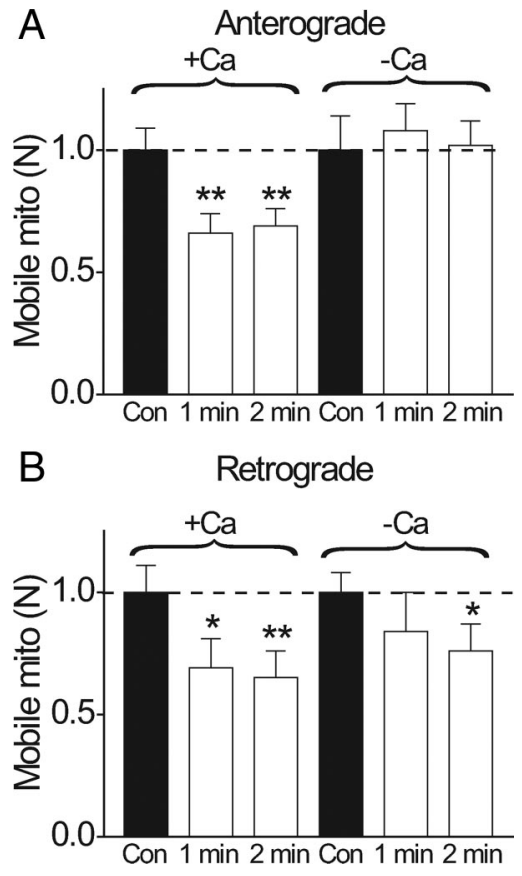

Figure 5. Activity-dependent reduction in number of mobile mitochondria is calciumdependent. Relative changes in the number of mobile mitochondria $(N)$ before and during repetitive stimulation (1 or $2 \mathrm{~min} ; 200 \mathrm{~Hz}$ ). $\boldsymbol{A}$, Anterograde direction. $\boldsymbol{B}$, Retrograde direction. In each experiment, the control consisted of counting $N$ for 1 or 2 min before stimulation. Stimulation was immediately applied $(200 \mathrm{~Hz})$, and $N$ was counted during stimulation in the same axon for the same time interval as in control (1 or $2 \mathrm{~min}$ ). Each nerve was stimulated only once. The graph shows the relative change in $N$ during stimulation compared to the control. In normal Ringer's solution, experiments were performed in 8 nodes for 1 min stimulations and 16 nodes in 2 min stimulations. In Ca-free conditions, the nerve was bathed in Ca-free solutions for $15 \mathrm{~min}$ before the experiment began. Notice that stimulation reduces anterograde $N$ to $66 \%$ (1 min; $N=8$ nodes) and $69 \%$ ( 2 min; $N=16$ nodes), and retrograde $N$ to $69 \%$ ( $1 \mathrm{~min}$ ) and $65 \%$ (2 $\mathrm{min})$. This activity-dependent reduction of $N$ was completely abolished in Ca-free solutions for anterograde movement $(\boldsymbol{A})$, which was slightly increased by $8 \%$ (1 min) and $2 \%$ (2 min) compared to prestimulation values. For the retrograde movement $(\boldsymbol{B})$, there was a statistically significant reduction for 2 min stimulation (N reduced to $76 \%$ ). ${ }^{*} p<0.05 ;{ }^{* *} p<0.01$. Error bars indicate SEM.

\section{Pause duration}

We simply surveyed all kymograph sections for a given node and measured all different pause durations, regardless of movement directions and whether the pauses came from the same or from different mitochondria.

It is critical to eliminate bias in the analysis. To this end, all data $(N, V$, and $P)$ in this paper were analyzed by one or two of us completely blinded to the conditions of the experiment. Furthermore, to confirm the reliability of the kymography analysis regarding $N$, we randomly selected several experiments and used a simple visual count of mobile mitochondria through a node by viewing repeated replay of the same movie. This visual count of $N$ corroborates with $N$ determined from the kymographs. The following kymographic results were from a total of 1544 mitochondria from 72 nodes.

\section{Number of mobile mitochondria was reduced during repetitive action potentials}

Figure 5 shows that the number of mobile mitochondria was reduced during stimulation ( 1 or $2 \mathrm{~min} ; 200 \mathrm{~Hz}$ ). The number of mobile mitochondria was counted during the stimulation period ( 1 or $2 \mathrm{~min}$ ) and compared with the number counted for the same period in the same node immediately before stimulation. 

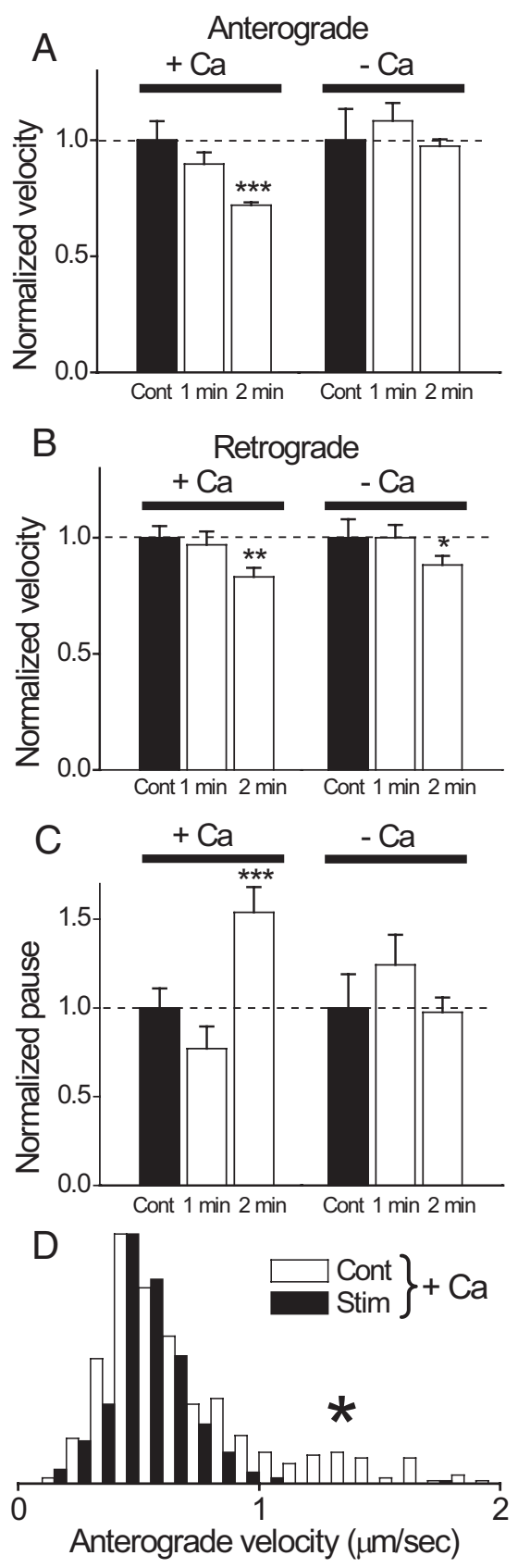

Figure 6. Activity-dependent changes in velocity and pause durations of mobile mitochondria are calcium dependent. The velocities and pause durations during stimulations were expressed relative to the control. Data are from the same set of nerves in Figure 5. A, Anterograde velocity. Normal Ca ( + Ca), Velocity was reduced by $10 \%$ (from $0.51 \pm 0.042 \mu \mathrm{m} / \mathrm{s}$ to $0.45 \pm$ $0.026 \mu \mathrm{m} / \mathrm{s} ; N=8$ nodes) during 1 min stimulation, and by $27 \%$ (from $0.73 \pm 0.02 \mu \mathrm{m} / \mathrm{s}$ to $0.53 \pm 0.01 \mu \mathrm{m} / \mathrm{s} ; N=16$ nodes) during 2 min stimulation. (a free $(-C a)$, Velocity was reduced by $9 \%$ (from $0.45 \pm 0.03 \mu \mathrm{m} / \mathrm{s}$ to $0.49 \pm 0.035 \mu \mathrm{m} / \mathrm{s} ; N=8$ nodes) during $1 \mathrm{~min}$ stimulation, and by $3 \%$ (from $0.54 \pm 0.02 \mu \mathrm{m} / \mathrm{s}$ to $0.52 \pm 0.02 \mu \mathrm{m} / \mathrm{s} ; N=8$ nodes) during 2 min stimulation. $\boldsymbol{B}$, Retrograde velocity. Normal $\mathrm{C}$, Velocity was reduced by $4 \%$ (from $0.46 \pm$ $0.026 \mu \mathrm{m} / \mathrm{s}$ to $0.46 \pm 0.02 \mu \mathrm{m} / \mathrm{s}$ ) during 1 min stimulation, and by $18 \%$ (from $0.69 \pm 0.027$ $\mu \mathrm{m} / \mathrm{s}$ to $0.57 \pm 0.026 \mu \mathrm{m} / \mathrm{s}$ ) during 2 min stimulation. Ca free, There was no change in velocity (from $0.46 \pm 0.03 \mu \mathrm{m} / \mathrm{s}$ to $0.46 \pm 0.026 \mu \mathrm{m} / \mathrm{s}$ ) during $1 \mathrm{~min}$ stimulation, and velocity was reduced by $11 \%$ (from $0.54 \pm 0.024 \mu \mathrm{m} / \mathrm{s}$ to $0.48 \pm 0.02 \mu \mathrm{m} / \mathrm{s}$ ) during $2 \mathrm{~min}$ stimulation. Numbers of the node are the same as in $\boldsymbol{A}$. C, Pause duration. Normal Ca, Pause duration was reduced by $23 \%$ (from $16.6 \pm 2.8 \mathrm{~s}$ to $12.8 \pm 2.1 \mathrm{~s}$ ) during 1 min stimulation and increased by $54 \%$ (from $12.3 \pm 0.9 \mathrm{~s}$ to $18.9 \pm 1.8 \mathrm{~s}$ ) during 2 min stimulation. Only the increase in 2 min stimulation is statistically significant. Ca free, Pause duration increased by $24 \%$ (from $12.6 \pm 2.3 \mathrm{~s}$ to $15.7 \pm 2.6 \mathrm{~s}$ ) during 1 min stimulation and was reduced by $2 \%$ (from $16.9 \pm 2.4$ s to $16.5 \pm 1.4$ s) during 2 min stimulation. Neither change is statistically significant.

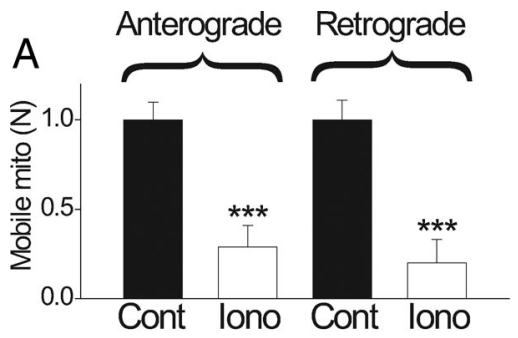

B

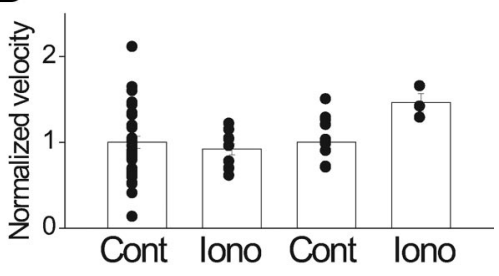

C

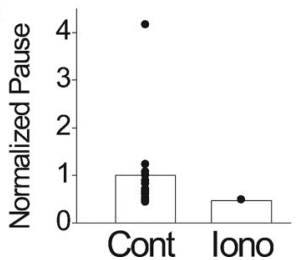

Figure 7. Effects of Ca ionophore on mitochondrial movement. Bath application of Ca ionophore ionomycin (10 $\mu \mathrm{m}$ ) was used to raise cytosolic Ca. Control, Zero Ca plus ionomycin for 15 min. Iono, Fifteen minutes after switching from control to high Ca ( $15 \mathrm{~mm}$ Ca plus ionomycin). $\boldsymbol{A}$, Number of mobile mitochondria decreased by $71 \%$ and $80 \%$, respectively, in anterograde and retrograde directions ( $N=6$ nodes) from 0 to high calcium. $B$, Velocity was reduced by $9 \%$ in anterograde (from $0.416 \pm 0.03 \mu \mathrm{m} / \mathrm{s}$ to $0.38 \pm 0.03 \mu \mathrm{m} / \mathrm{s} ; p>0.05$ ) and increased by $47 \%$ in retrograde (from $0.44 \pm 0.03 \mu \mathrm{m} / \mathrm{s}$ t $00.65 \pm 0.05 \mu \mathrm{m} / \mathrm{s} ; p>0.05$ ). C, Pause duration was reduced by $50 \%$ (from $14.78 \pm 3.5$ s to $7.36 \pm 0.0 \mathrm{~s} ; p<0.01$ ). Changes in $\boldsymbol{B}$ and $\boldsymbol{C}$ are not considered significant because of the small number of mobile mitochondria in high Ca (lono). ${ }^{* * *} p<0.005$. Error bars indicate SEM.

During stimulation, the number of mobile mitochondria was reduced to $66 \%$ ( $1 \mathrm{~min}$ ) and $69 \%$ (2 $\mathrm{min}$ ) for anterograde movement (Fig. 5A), and to 69\% (1 $\mathrm{min})$ and 65\% (2 min) for retrograde movement (Fig. 5B). Recovery from stimulation was measured from the same node under quiescent conditions $5 \mathrm{~min}$ after stimulation. For anterograde movement, $N$ recovered from $65 \%$ to $92 \%$ after 1 min stimulations, and from $69 \%$ to $80 \%$ after 2 min stimulations (data not shown). Interestingly, no recovery was seen for retrograde movement, at least at 5 min after stimulation. Our choice of 1-2 min stimulation at $200 \mathrm{~Hz}$ is within the operating range of peripheral nerves in previous studies [e.g., 60 min, intermittent stimulation at $50-200 \mathrm{~Hz}$ in metabolic studies (Connelly, 1959); $30 \mathrm{~min}$ at $90 \mathrm{~Hz}$ continuous stimulation in intact animals (Mima et al., 2004)].

\section{Activity-dependent reduction in $\mathrm{N}$ was $\mathrm{Ca}$ dependent}

To examine whether the reduction in $N$ during action potentials was caused by the evoked Ca increase, we performed stimulations in Ca-free solutions. We began experiments 15 min after perfusing the bath with a Ca-free solution, at which time the evoked

\section{$\leftarrow$}

Numbers of nodes are the same as in $\boldsymbol{A} .{ }^{*} p<0.05 ;{ }^{* *} p<0.01$; ${ }^{* * *} p<0.005 . \boldsymbol{D}$, Histogram of anterograde velocity shows that the mitochondrial population with the most rapid velocity (asterisk) is preferentially reduced by 2 min stimulation ( $N=8$ nodes) in normal Ringer's solution. The histogram is plotted as a probability density function (normalized so that the area is 1) to facilitate comparison of the shapes of the two distributions. Error bars indicate SEM. 
calcium response was abolished (Fig. $2 \mathrm{~A}$ ). This completely prevented action potentials from reducing $N$ for the anterograde direction (Fig. 5A). Interestingly, some activity-dependent reduction of $N$ in the retrograde direction still persisted in $\mathrm{Ca}$-free conditions (Fig. 5B).

\section{Activity-dependent modulation of velocity and pause duration}

Is the effect of action potential restricted only to reducing $N$ ? Do action potentials modulate dynamics $(V$ and $P$ ) of mobile mitochondria? To examine this, we analyzed $V$ and $P$ of mobile mitochondria during repetitive action potentials. Figure $6, A$ and $B$, shows that there is an activity-dependent reduction of velocity in normal Ringer's solution that becomes statistically significant during 2 min stimulations. Thus, during 2 min stimulations, the velocity is reduced to $73 \%$ of control for anterograde movement (from $0.73 \pm 0.02 \mu \mathrm{m} / \mathrm{s}$ to $0.53 \pm 0.01 \mu \mathrm{m} / \mathrm{s} ; N=16$ nodes) and to $82 \%$ of control for retrograde movement (from $0.69 \pm 0.027$ $\mu \mathrm{m} / \mathrm{s}$ to $0.57 \pm 0.026 \mu \mathrm{m} / \mathrm{s} ; N=16$ nodes). Interestingly, histogram analysis shows that for anterograde movement, the activitydependent reduction in velocity during 2 min stimulation occurs predominantly in the most rapid mitochondrial population (Fig. $6 D$, asterisk). When stimulated in Ca-free solutions, activitydependent reduction in velocity was abolished for anterograde, but not for retrograde movement (Fig. 6B) (2 min stimulation in Ca-free solution).

In addition to reduction in mitochondrial velocity, stimulation also leads to an increase in pause duration for mitochondrial movement through a node. Figure $6 C$ shows that the pause duration of mitochondrial movement was increased by $\sim 54 \%$ (from control of $12.3 \pm 0.9 \mathrm{~s}$ to $18.9 \pm 1.8 \mathrm{~s} ; N=16$ nodes; $p<$ 0.005 ) during a $2 \mathrm{~min}$ stimulation. There is no statistically significant increase in pause duration when nerves were stimulated in Ca-free solutions (Fig. 6C).

Collectively, the results of Figures 5 and 6 demonstrate that repetitive action potentials retard mitochondrial motility through a node by three means: reduction of number of mobile mitochondria, reduction in velocity, and increase in pause duration. This effect is Ca dependent, particularly in the anterograde direction.

\section{Ca ionophore}

To directly examine the effect of raising cytosolic Ca on mitochondrial motility, we raised the bath $\mathrm{Ca}$ from 0 to $15 \mathrm{~mm}$ after permeabilizing the plasma membrane with a Ca ionophore (10 $\mu \mathrm{M}$ ionomycin). Furthermore, we coapplied $2 \mu \mathrm{M}$ thapsigargin to block Ca uptake into the ER (by blocking the ATP using Ca pump SERCA) as cytosolic Ca is raised. The rationale is that ER calcium uptake might cause a local ATP depletion near mitochondria, which will de-energize the motors and reduce mitochondrial movement unrelated to a direct effect of $\mathrm{Ca}$ on the movement. Blocking ER calcium uptake with thapsigargin will thus reveal any direct effect of calcium on mitochondrial motility. Figure $7 \mathrm{~A}$ shows that Ca ionophore (labeled Iono) dramatically reduces $N$ to $29 \%$ (anterograde) and $20 \%$ (retrograde) of control values. There was no significant effect on the velocity (Fig. $7 B$ ) and pause duration (Fig. 7C). This inhibitory effect of Ca ionophore on $\mathrm{N}$ is partially reversible: switching the bath from 15 to $0 \mathrm{~mm} \mathrm{Ca}$ (in the presence of Ca ionophore) restores $N$ to $70 \%$ and $55 \%$, respectively, for anterograde and retrograde directions (data not shown). The absolute cytosolic Ca elevation was not measured in these studies, but it is most certainly higher than that achieved during action potential stimulations.

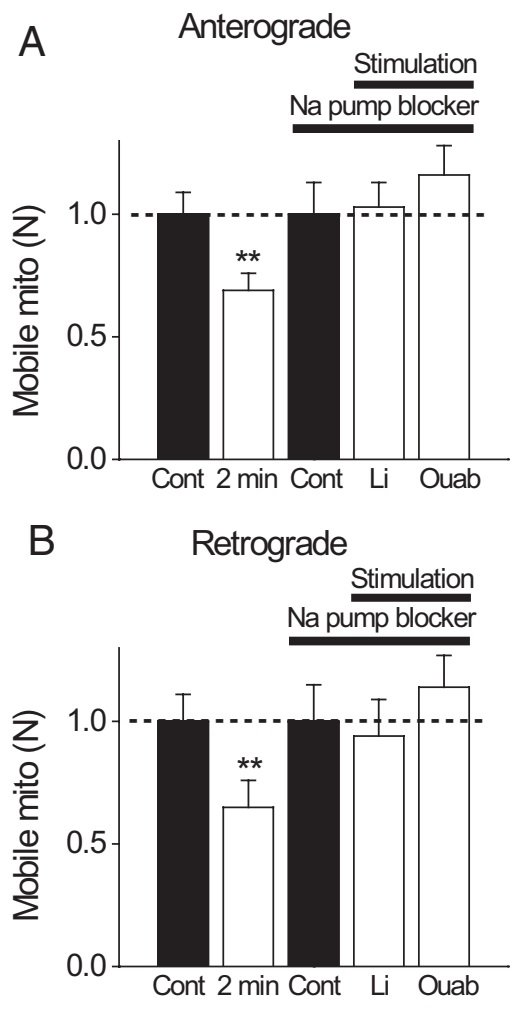

Figure 8. $\quad \boldsymbol{A}, \boldsymbol{B}$, Activity-dependent reduction in number of mobile mitochondria is $\mathrm{Na} / \mathrm{K}$ ATPase dependent. Relative changes in the number of mobile mitochondria before [control (Cont)] and during repetitive stimulation ( $2 \mathrm{~min} ; 200 \mathrm{~Hz}$ ). Nerves were stimulated either in normal Ringer's solution or in two modified Ringer's solutions designed to block Na/K-ATPase but preserve action potentials [lithium replacing all bath $\mathrm{Na}$ ( $N=6$ nodes) or $0.5 \mathrm{~mm}$ ouabain (Ouab) in Ringer's solution ( $N=6$ nodes)]. Note that blocking Na/K-ATPase completely blocks activity-dependent reduction in the number of mobile mitochondria. ${ }^{* *} p<0.01$. Error bars indicate SEM.

\section{Blocking Na/K-ATPase blocks activity-dependent retardation of mitochondria}

To examine whether activation of $\mathrm{Na} / \mathrm{K}$-ATPase plays a role in regulation of mitochondrial motility, we blocked the $\mathrm{Na} / \mathrm{K}$ ATPase with ouabain or lithium (Gordon et al., 1990) during action potentials. Nerves were incubated in a calcium-containing Ringer's solution with ouabain $(0.5 \mathrm{~mm})$ or with $\mathrm{Na}$ replaced by Li for $15 \mathrm{~min}$ before the experiments began. Ouabain $(0.5 \mathrm{~mm})$ and $\mathrm{Li}$ (which is permeable through $\mathrm{Na}$ channels) did not affect the compound action potentials and only have minor effects on the resting mobility of mitochondria before stimulation (resting $N$ values are $89 \%$ and $108 \%$ of control for anterograde and retrograde movements in lithium, respectively, and $96 \%$ and $120 \%$ of the control for anterograde and retrograde movements in ouabain, respectively). Strikingly, activity-dependent retardation of mitochondria was completely abolished by Na-pump blockers during 2 min stimulations. Figure 8 shows that in lithium or ouabain, action potentials failed to reduce the number of mobile mitochondria for anterograde $(A)$ and retrograde $(B)$ directions ( 2 min stimulation data in normal Ringer's solution is shown for comparison). Furthermore, activity-dependent reduction in velocities (Fig. 9A, $B$ ) and prolongation of pause duration $(C)$ were also mostly abolished when the Na pumps were blocked. These studies suggest that activation of $\mathrm{Na} / \mathrm{K}$-ATPase during nerve activity retards mitochondrial motility. 

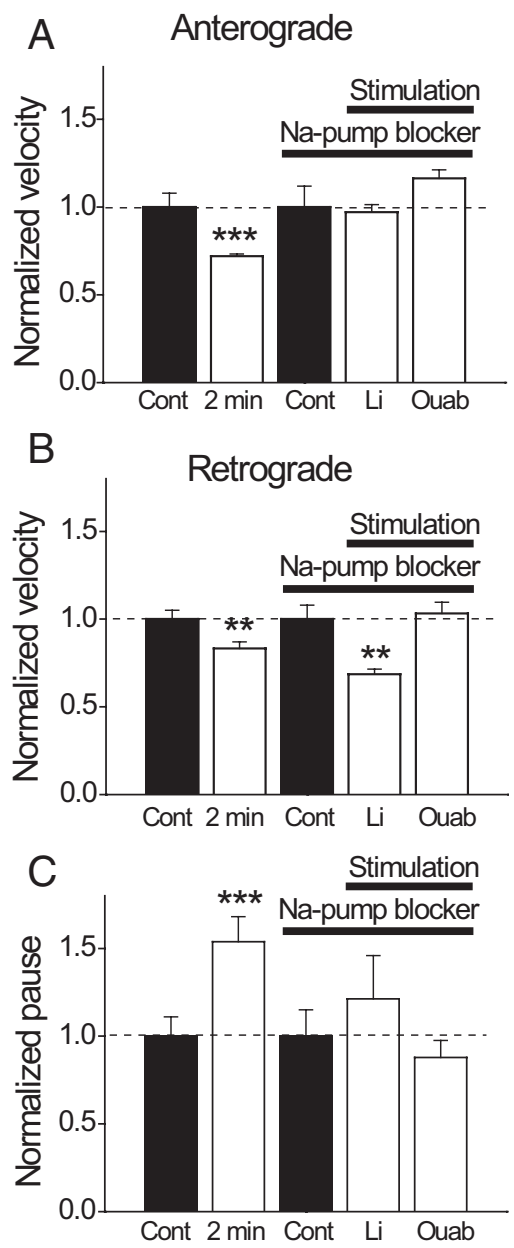

Figure 9. $\quad A-C$, Activity-dependent changes in velocity and pause durations of mobile mitochondria is $\mathrm{Na} / \mathrm{K}$-ATPase dependent. Relative changes in these parameters before (Cont) and during stimulation ( $2 \mathrm{~min} ; 200 \mathrm{~Hz}$ ) in either normal or modified Ringer's solution [Li, $N=6 ; 0.5$ mм ouabain (Ouab), $N=6$ ] in which $\mathrm{Na} / \mathrm{K}$-ATPase was blocked but action potentials preserved. Note that blocking $\mathrm{Na} / \mathrm{K}$-ATPase in most cases blocked activity-dependent changes in velocity and pause durations, except in the case of retrograde movement in lithium. ${ }^{* *} p<0.01$; ${ }^{* * *} p<0.005$. Error bars indicate SEM.

\section{Artificial stimulation of Na/K-ATPase retards mitochondrial motility}

To directly confirm that activation of Na/K-ATPase retards mitochondrial motility, we artificially stimulated the $\mathrm{Na} / \mathrm{K}$ ATPase by bath application of a Ca-free mixture containing 10 $\mu \mathrm{M}$ veratridine (a sodium channel opener to elevate intracellular $\mathrm{Na}$ ) and elevated extracellular $\mathrm{K}$ (from 3 to $20 \mathrm{~mm}$ ). The Ca-free condition is important to ensure that any effects on mitochondrial motility produced by this mixture cannot be ascribed to calcium. This mixture dramatically reduced the number of mobile mitochondria to $19 \%$ and $12 \%$ of control values for the anterograde and retrograde directions, respectively (Fig. 10A), without significantly affecting the velocities $(B)$ and the pause duration $(C)$. To verify that mitochondrial immobilization is caused by $\mathrm{Na} / \mathrm{K}$-ATPase activation, we blocked the $\mathrm{Na} / \mathrm{K}$-ATPase with ouabain $(0.5 \mathrm{~mm})$ during the mixture application. Ouabain dramatically prevented the mixture from reducing the number of mobile mitochondria (Fig. 10A), proving that the mitochondrial immobilization is caused by $\mathrm{Na} / \mathrm{K}$-ATPase activation.

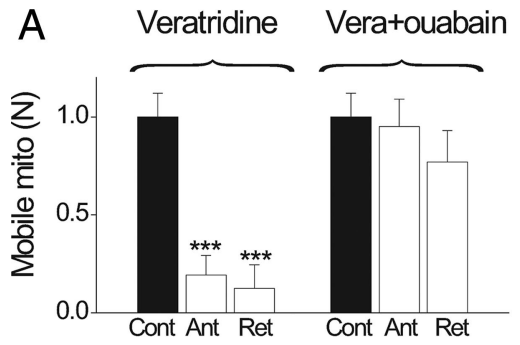

B
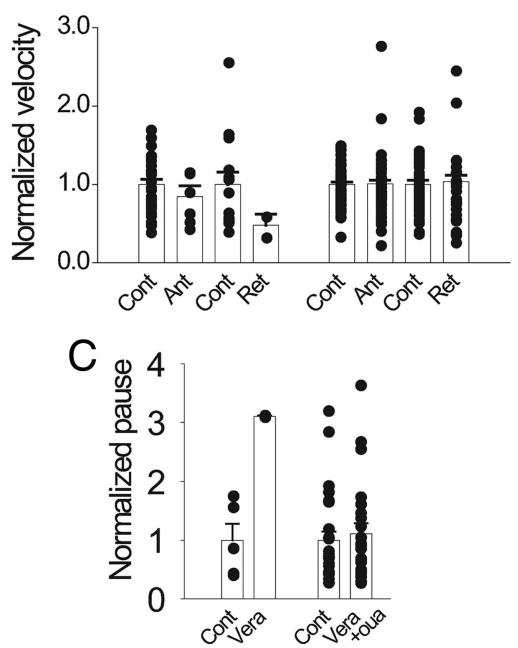

Figure 10. Artificial activation of Na/K-ATPase blocks mitochondrial movement. Nerves were incubated in a Ca-free solution for 15 min before switching to a Ca-free bath mixture designed to artificially active $\mathrm{Na} / \mathrm{K}$-ATPase [intracellular $\mathrm{Na}$ raised by $10 \mu \mathrm{m}$ veratridine (Vera); extracellular $\mathrm{K}$ raised from 3 to $20 \mathrm{~mm}$ ]. Plots show results relative to the control in the $\mathrm{Na} / \mathrm{K}$ ATPase-activating mixture. $\boldsymbol{A}$, Artificial activation of $\mathrm{Na} / \mathrm{K}$-ATPase dramatically reduced the number of mobile mitochondria by $81 \%$ in anterograde (Ant) and $88 \%$ in retrograde (Ret; $N=$ 6 nodes). This reduction was mostly prevented by ouabain (oua; $0.5 \mathrm{~mm}$ ) coapplication $(N=6$ nodes). ${ }^{* *} p<0.005 . B, C$, Activation of Na/K-ATPase had no significant effect on the velocity and pause durations. Error bars indicate SEM.

The effect of manipulation of Na pumps on mitochondrial motility is not attributable to secondary effects on intra-axonal Ca

Given the strength of direct molecular evidence for regulation of mitochondrial motility through elevated $\mathrm{Ca}$, an important issue is whether pharmacological manipulation of $\mathrm{Na}$ pumps (ouabain, lithium, and veratridine) affects mitochondrial motility because of secondary effects on intra-axonal $\mathrm{Ca}$. We therefore examined possible effects of these Na-pump modifiers on intra-axonal $\mathrm{Ca}$ at the node of Ranvier. First, we examined the effects of ouabain and lithium on the intra-axonal $\mathrm{Ca}$ response evoked by action potentials at different stimulation frequencies. Frequency dependence of the evoked Ca response was measured for each drug according to the paradigm of Figure $1 D$. A control was first measured in normal Ringer's solution, followed by a measurement after $15 \mathrm{~min}$ of drug treatment in the same fiber. The result was expressed relative to the maximal response $(200 \mathrm{~Hz})$ in the control Ringer's solution for each respective drug. The results (Fig. 11) show that ouabain $(A)$ and lithium $(B)$ produced no significant change in the frequency dependence of evoked intra-axonal $\mathrm{Ca}$ response at the node. These frequency-dependence curves were generated with brief pulses. Figure $11 \mathrm{C}$ shows that for long-duration pulses $(2 \mathrm{~min})$ there is no change in the shape of the evoked Ca response in the presence of ouabain or lithium. Second, we examined veratridine. In the motility experiments of Figure 10, we used veratridine to artificially activate 

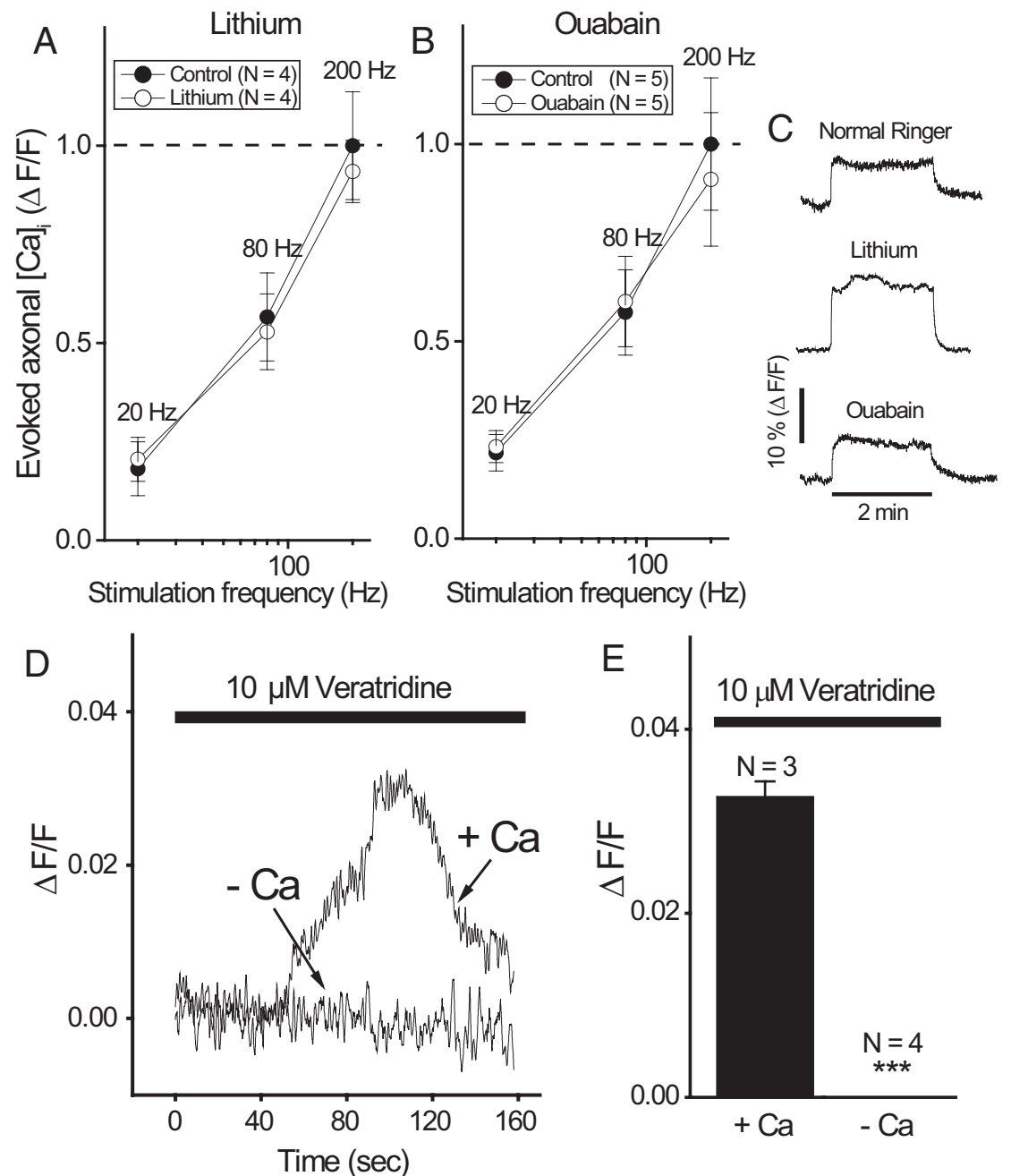

Figure 11. Manipulation of $\mathrm{Na} / \mathrm{K}$-ATPase does not cause secondary changes in intra-axonal $\mathrm{C} a . A, B$, Frequency dependence of evoked Ca axonal response in ouabain $(\boldsymbol{A})$ and lithium $(\boldsymbol{B})$. In each panel, the frequency dependence was first measured in the control (i.e., normal Ringer's solution), then measured again after 15 min of drug application in the same fiber. The evoked Ca responses were normalized relative to the peak response $(200 \mathrm{~Hz})$ in the control. The paradigm for measurement of evoked Ca response is similar to that in Figure $1 D$. The frequency dependence of evoked Ca response generated by briefpulses (4s duration) is shown. $C$, Shape of evoked Ca response is similar in normal $(N=3)$, lithium $(N=2)$, and ouabain $(N=2)$ during prolonged stimulation (2 min duration). Representative traces are shown here. $\boldsymbol{D}$, Effects of veratridine plus $20 \mathrm{~mm} \mathrm{~K}$ on intra-axonal Ca in normal Ringer's and in Ca-free bath solutions. $\boldsymbol{E}$, Averaged results of the peak Ca response from $\boldsymbol{D}$. ${ }^{* *} p<0.005$. Error bars indicate SEM.

the Na pumps in Ca-free solutions to show that Na-pump activation led to retardation of mitochondrial motility independent of intraaxonal Ca elevation. However, it remains possible that veratridine can still cause intracellular Ca release from mitochondria or ER under Ca-free conditions. We therefore directly measured intra-axonal Ca during veratridine treatment, in normal and in Ca-free bath solutions. In normal solutions (Ca present in the bath), veratridine caused the expected elevation in intra-axonal Ca (Fig. 11D). However, veratridine no longer caused elevation in intra-axonal Ca when applied in Ca-free bath solutions (Fig. 11D,E). This demonstrates that the retardation in mitochondrial motility induced by veratridine in Ca-free solutions (Fig. 10) cannot be explained by intraaxonal Ca elevation. Collectively, Figure 11 shows that the effects of Na pump manipulation on mitochondrial motility in Figures 8-10 cannot be explained by secondary effects on intra-axonal Ca.

\section{Blocking cytoskeleton systems immobilizes mitochondria}

Mitochondria are thought to use both the microtubule and microfilament (actin/myosin-based transport) systems for move- ment. To examine the role of these two cytoskeleton systems in mitochondrial motility in myelinated axons, we compared mitochondrial motility in nerves taken from the same frog with or without a $2 \mathrm{~h}$ incubation with nocodazole (to disrupt the microtubule network; $50 \mu \mathrm{M}$; $N=6$ nodes) or cytochalasin $\mathrm{D}$ (to disrupt the actin network; $50 \mu \mathrm{g} / \mathrm{ml} ; N=6$ nodes). Both agents resulted in a significant reduction in the number of mobile mitochondria ( $N$ reduced by $66 \%$ and $83 \%$ in anterograde and retrograde directions, respectively, for nocodazole; $N$ reduced by $32 \%$ and $71 \%$ in anterograde and retrograde directions, respectively, for cytochala$\sin$ D) without significant effects on the velocities and pause durations.

\section{Discussion}

This work produces the first spatial resolution of nodal calcium signaling and shows that activity-dependent calcium elevation retards nodal mitochondrial motility in a manner similar to that reported for synapses. In addition, we have produced the first demonstration that physiologic activation of $\mathrm{Na}$ pumps retards mitochondrial motility, a novel feature in mitochondrial dynamics that might be unique to the node of Ranvier.

\section{Action potentials evoke localized Ca signaling at PNS nodes of Ranvier} Given the exquisite nodal specialization of ionic channels in myelinated nerves (Chiu and Ritchie, 1980; Poliak and Peles, 2003), the first study to examine axonal calcium signaling in CNS myelinated fibers yielded a surprise (Zhang et al., 2006). Instead of localized nodal calcium signaling, activity-evoked calcium signaling in CNS axons is spatially uniform and bears no spatial relationship to myelination. In striking contrast to the CNS, this work shows that Ca signaling in PNS myelinated axons is highly localized to the node of Ranvier. Intriguingly, this is paralleled by a marked difference in axonal mitochondrial distribution in these two fiber types. Axonal mitochondrial density is increased at the nodal region in PNS (Fabricius et al., 1993), but is uniform in nodes and internodes in the CNS (Edgar et al., 2008). Our study suggests that this differential mitochondrial distribution is caused by a marked difference in spatial Ca signaling: discrete nodal Ca signaling in the PNS recruits mitochondria to the nodal region, whereas uniform nodal and internodal Ca signaling in the CNS produces uniform mitochondrial distribution. It is tempting to speculate that internodal calcium signaling in the CNS but not the PNS makes the myelinated axons of the former more prone to excitotoxic damage than those of the latter.

\section{Action potentials regulate mitochondrial motility}

Studies of mitochondrial motility in myelinated axons began in the 1970s using light microscopy (Cooper and Smith, 1974), and 
recently involved sophisticated transgenic mice expressing mitochondrial markers (Misgeld et al., 2007). Despite these advances, regulation of nodal mitochondrial movement by action potentials has not been studied. Our study demonstrated that mitochondria motility is retarded by action potentials via at least two mechanisms: a calcium-dependent and an Na-pump-dependent mechanism. The calcium-dependent mechanism likely involves a direct inhibitory action of calcium on an adaptor protein that controls mitochondrial motors, as already described in the synapse (Yi et al., 2004; Saotome et al., 2008; Cai and Sheng, 2009; Macaskill et al., 2009; Wang and Schwarz, 2009). The second mechanism involving regulation by physiologic activation of the Na pump is novel. At present, the signaling pathway that couples Na-pump activation to retardation of mitochondrial motility remains unclear. One clear possibility is local ATP depletion after Na-pump activation. Alternatively, mitochondrial motors could be stopped by an increase in ADP (Mironov, 2007). Finally, we cannot rule out a yet-to-be-identified direct coupling between $\mathrm{Na}$ pumps and mitochondria.

\section{Coupling between $\mathrm{Na}$-pumps and mitochondrial movement may be unique for the node of Ranvier}

Compared with the synapse, regulation of mitochondria motility by $\mathrm{Na}$ pumps might be particularly important at the node of Ranvier because of a marked difference in ionic signaling mode and unique geometry.

First, unlike the synapse where the neurotransmittermediated Ca influx is very high, the evoked Ca response in a node is probably much smaller. In fact, some nodes in this study barely showed a detectable Ca response. This suggests that in a node, calcium signaling alone may not be strong enough to recruit mitochondria compared to a synapse where calcium influx is large. Hence, an extra recruitment mechanism mediated by $\mathrm{Na}$ pumps might be needed. Second, given its high Na channel density (Chiu, 1980) and a large surface-to-volume ratio attributable to nodal constriction, the node of Ranvier is geometrically unique to allow robust intracellular $\mathrm{Na}$ signaling to activate $\mathrm{Na}$ pumps to arrest mitochondria. Repetitive nerve activity has been shown to cause significant activation of the $\mathrm{Na} / \mathrm{K}$-ATPase in myelinated axons (Gordon et al., 1990). Hence, it is tempting to generalize that any system that relies heavily on $\mathrm{Na}$ signaling (the node being the prime example) might use Na-pump activation, rather than calcium, to translate nerve activity to mitochondrial movement modulation.

Granted this, however, there are several intriguing issues that require additional studies.

\section{Synergism between $\mathrm{Ca}$ and $\mathrm{Na} / \mathrm{K}$-ATPase?}

Why should $\mathrm{Ca}$-free solution and blockage of $\mathrm{Na} / \mathrm{K}$-ATPase, each by itself, be so effective in blocking activity-dependent retardation of mitochondrial motility, particularly in the anterograde direction (compare Figs. 5, 8)? One possibility is synergism. For example, Ca elevation may draw mitochondria axially toward the plasma membrane, but because of a weak calcium rise, this by itself is not sufficient to arrest the mitochondria. However, once the mitochondria are drawn near the plasma membrane, local depletion of ATP created by $\mathrm{Na} / \mathrm{K}$-ATPase activity might act as the final cause for arresting mitochondria. This synergistic scenario would explain why blocking either component in this scheme $(\mathrm{Na} / \mathrm{K}-\mathrm{ATP}$ ase or $\mathrm{Ca}$ rise) prevents action potentials from stopping mitochondria. When Ca rise is blocked, mitochondria are no longer drawn toward the plasma membrane to interact with the $\mathrm{Na} / \mathrm{K}$-ATPase. When $\mathrm{Na} / \mathrm{K}$-ATPase is blocked, it abolishes the micro-ATP domain that finally arrests the mitochondria initially drawn toward the membrane by calcium influx. Although speculative, some features of this scheme are possible. In cultured cells, Ca influx has been shown to induce axial movement of mitochondria toward the plasma membrane (Kolikova et al., 2006; Quintana et al., 2006). Furthermore, microdomains of ATP are known to exist in some systems (Kennedy et al., 1999), and microdomains of ATP might exist underneath the nodal membrane to interact with mitochondria drawn to its proximity. Our present study resolves only longitudinal movement of mitochondria, and high-resolution images of axial movement of mitochondria in the nodal region are needed to resolve this issue.

\section{Normal and pathological implications}

Action potentials reduced the number of mobile mitochondria at a node of Ranvier by $\sim 40 \%$. Assuming a corresponding number of mitochondria gets recruited to the node, what is the function of this recruitment? First, mitochondria might be recruited to the node to match energy demands during intense stimulation. However, this mitochondrial recruitment constitutes only a small fraction of the total mitochondria, since approximately two-thirds are already immobile and unaffected by action potentials. On the other hand, mitochondria might be highly heterogeneous in their metabolic activity, and not all are metabolically useful to supply energy to the node. Mobile mitochondria might be the most metabolically active, and harvesting them is the best strategy to match energy needs to nerve activity. Indeed, our data suggest that the most rapid mitochondria are preferentially arrested by action potentials (Fig. $6 D$, asterisk). Second, recruiting mitochondria to active nodes of Ranvier might serve to increase calcium buffering at that site. Mitochondria can take up plasmalemmal Ca influx, locally reducing Ca concentration. Third, activity-dependent immobilization of mitochondria might contribute to long-term patterning of mitochondria along myelinated axons, influencing spatial signaling of molecules such as reactive oxygen species with chronic biological impact. In our study, it is of interest that activity-dependent retardation of mitochondria shows only partial (anterograde) or no (retrograde) recovery when measured $5 \mathrm{~min}$ after cessation of activity. This raises the intriguing issue whether prolonged, intermittent nerve activity during development might contribute to permanent mitochondrial patterning. For example, are the immobile mitochondria (approximately two-thirds of total) in quiescent nerves caused by intermittent nerve activity during development?

Finally, our findings have pathophysiological implications for multiple sclerosis. Errors in activity-dependent immobilization of mitochondria may contribute to axonal pathology. Toxic wastes (free radicals) from highly mobile mitochondria may have less chance to linger to damage axons. Thus, although activitydependent immobilization of mitochondria may serve regional energy demands, excessive immobilization in inappropriate and vulnerable sites may be injurious. The known patterns of abnormal hyperexcitable nerve activity in multiple sclerosis (Smith and McDonald, 1980; Smith and McDonald, 1999) may overretard mitochondria with damaging results. For example, in various animal models of dysmyelination, the reported inappropriate immobilization of mitochondria in the nodal region (Einheber et al., 2006) or the increased mitochondrial density in dysmyelinated regions (Andrews et al., 2006) may arise from excessive overrecruitment of mitochondria by action potentials. Furthermore, excessive retardation of mitochondria may occur because of inappropriately high expression of $\mathrm{Na}_{\mathrm{v}} 1.6$ in demyelinated 
axons (Craner et al., 2004; Waxman, 2006). The persistent component of $\mathrm{Na}_{\mathrm{v}} 1.6$, according to our hypothesis, will exacerbate $\mathrm{Na}$ influx and augment Na-pump-mediated stoppage of mitochondria. Errors in activity-dependent mitochondrial recruitment also might occur in multiple sclerosis because of a change in $\mathrm{Na}$ /K-ATPase expression (Young et al., 2008; Mahad et al., 2009), or a change in internal structure of the axon that affects the micro-ATP domain that couples $\mathrm{Na} / \mathrm{K}$-ATPase to mitochondrial motility. Excessive Na influx has been linked to axonal pathology, and $\mathrm{Na} / \mathrm{K}$-ATPase has been implicated, although the manner of involvement is unclear (Bechtold et al., 2005). It is of interest that ouabain has been shown to improve conduction in demyelination (Kaji and Sumner, 1989), raising the intriguing issue of whether excessive coupling of $\mathrm{Na} / \mathrm{K}$-ATPase to mitochondria occurs in multiple sclerosis, and whether blocking this excessive coupling has long-term benefits. Future studies need to examine possible defects in activity-dependent retardation of mitochondria in animal models of multiple sclerosis.

\section{References}

Andrews H, White K, Thomson C, Edgar J, Bates D, Griffiths I, Turnbull D, Nichols P (2006) Increased axonal mitochondrial activity as an adaptation to myelin deficiency in the Shiverer mouse. J Neurosci Res 83:1533-1539.

Andrews HE, Nichols PP, Bates D, Turnbull DM (2005) Mitochondrial dysfunction plays a key role in progressive axonal loss in multiple sclerosis. Med Hypotheses 64:669-677.

Baloh RH (2008) Mitochondrial dynamics and peripheral neuropathy. Neuroscientist 14:12-18.

Baloh RH, Schmidt RE, Pestronk A, Milbrandt J (2007) Altered axonal mitochondrial transport in the pathogenesis of Charcot-Marie-Tooth disease from mitofusin 2 mutations. J Neurosci 27:422-430.

Baron KT, Thayer SA (1997) CGP37157 modulates mitochondrial $\mathrm{Ca}^{2+}$ homeostasis in cultured rat dorsal root ganglion neurons. Eur J Pharmacol 340:295-300.

Bechtold DA, Yue X, Evans RM, Davies M, Gregson NA, Smith KJ (2005) Axonal protection in experimental autoimmune neuritis by the sodium channel blocking agent flecainide. Brain 128:18-28.

Berthold CH (1978) Morphology of normal peripheral axons. In: Physiology and pathobiology of axons (Waxman SG, ed), pp 3-63. New York: Raven.

Brough D, Schell MJ, Irvine RF (2005) Agonist-induced regulation of mitochondrial and endoplasmic reticulum motility. Biochem J 392:291-297.

Cai Q, Sheng ZH (2009) Moving or stopping mitochondria: Miro as a traffic cop by sensing calcium. Neuron 61:493-496.

Chan DC (2007) Mitochondrial dynamics in disease. N Engl J Med 356:1707-1709.

Chiu SY (1980) Asymmetry currents in the mammalian myelinated nerve. J Physiol 309:499-519.

Chiu SY, Ritchie JM (1980) Potassium channels in nodal and internodal axonal membrane of mammalian myelinated fibres. Nature 284:170-171.

Connelly CM (1959) Recovery processes and metabolism of nerve. Rev Mod Phys 31:475-484.

Cooper PD, Smith RS (1974) The movement of optically detectable organelles in myelinated axons of Xenopus laevis. J Physiol 242:77-97.

Cox DA, Conforti L, Sperelakis N, Matlib MA (1993) Selectivity of inhibition of $\mathrm{Na}(+)-\mathrm{Ca} 2+$ exchange of heart mitochondria by benzothiazepine CGP-37157. J Cardiovasc Pharmacol 21:595-599.

Craner MJ, Hains BC, Lo AC, Black JA, Waxman SG (2004) Co-localization of sodium channel Navl.6 and the sodium-calcium exchanger at sites of axonal injury in the spinal cord in EAE. Brain 127:294-303.

David G (1999) Mitochondrial clearance of cytosolic $\mathrm{Ca}^{2+}$ in stimulated lizard motor nerve terminals proceeds without progressive elevation of mitochondrial matrix $\left[\mathrm{Ca}^{2+}\right]$. J Neurosci 19:7495-7506.

Detmer SA, Chan DC (2007) Functions and dysfunctions of mitochondrial dynamics. Nat Rev Mol Cell Biol 8:870-879.

Dutta R, McDonough J, Yin X, Peterson J, Chang A, Torres T, Gudz T, Macklin WB, Lewis DA, Fox RJ, Rudick R, Mirnics K, Trapp BD (2006) Mitochondrial dysfunction as a cause of axonal degeneration in multiple sclerosis patients. Ann Neurol 59:478-489.
Edgar JM, McCulloch MC, Thomson CE, Griffiths IR (2008) Distribution of mitochondria along small-diameter myelinated central nervous system axons. J Neurosci Res 86:2250-2257.

Einheber S, Bhat MA, Salzer JL (2006) Disrupted Axo-glial junctions result in accumulation of abnormal mitochondria at nodes of Ranvier. Neuron Glia Biol 2:165-174.

Fabricius C, Berthold CH, Rydmark M (1993) Axoplasmic organelles at nodes of Ranvier. II. Occurrence and distribution in large myelinated spinal cord axons of the adult cat. J Neurocytol 22:941-954.

Gordon TR, Kocsis JD, Waxman SG (1990) Electrogenic pump (Na+/ $\mathrm{K}(+)$-ATPase) activity in rat optic nerve. Neuroscience 37:829-837.

Hollenbeck PJ, Saxton WM (2005) The axonal transport of mitochondria. J Cell Sci 118:5411-5419.

Hom JR, Gewandter JS, Michael L, Sheu SS, Yoon Y (2007) Thapsigargin induces biphasic fragmentation of mitochondria through calciummediated mitochondrial fission and apoptosis. J Cell Physiol 212:498508

Kaji R, Sumner AJ (1989) Effect of digitalis on central demyelinative conduction block in vivo. Ann Neurol 25:159-165.

Kennedy HJ, Pouli AE, Ainscow EK, Jouaville LS, Rizzuto R, Rutter GA (1999) Glucose generates sub-plasma membrane ATP microdomains in single islet beta-cells. Potential role for strategically located mitochondria. J Biol Chem 274:13281-13291.

Kolikova J, Afzalov R, Giniatullina A, Surin A, Giniatullin R, Khiroug L (2006) Calcium-dependent trapping of mitochondria near plasma membrane in stimulated astrocytes. Brain Cell Biol 35:75-86.

Macaskill AF, Rinholm JE, Twelvetrees AE, Rancibia-Carcamo IL, Muir J, Fransson A, Aspenstrom P, Attwell D, Kittler JT (2009) Mirol is a calcium sensor for glutamate receptor-dependent localization of mitochondria at synapses. Neuron 61:541-555.

Mahad D, Ziabreva I, Lassmann H, Turnbull D (2008) Mitochondrial defects in acute multiple sclerosis lesions. Brain 131:1722-1735.

Mahad DJ, Ziabreva I, Campbell G, Lax N, White K, Hanson PS, Lassmann H, Turnbull DM (2009) Mitochondrial changes within axons in multiple sclerosis. Brain 132:1161-1174.

Mima T, Oga T, Rothwell J, Satow T, Yamamoto J, Toma K, Fukuyama H, Shibasaki H, Nagamine T (2004) Short-term high-frequency transcutaneous electrical nerve stimulation decreases human motor cortex excitability. Neurosci Lett 355:85-88.

Mironov SL (2006) Spontaneous and evoked neuronal activities regulate movements of single neuronal mitochondria. Synapse 59:403-411.

Mironov SL (2007) ADP regulates movements of mitochondria in neurons. Biophys J 92:2944-2952.

Misgeld T, Kerschensteiner M, Bareyre FM, Burgess RW, Lichtman JW (2007) Imaging axonal transport of mitochondria in vivo. Nat Methods 4:559-561.

Pitter JG, Maechler P, Wollheim CB, Spat A (2002) Mitochondria respond to $\mathrm{Ca} 2+$ already in the submicromolar range: correlation with redox state. Cell Calcium 31:97-104

Poliak S, Peles E (2003) The local differentiation of myelinated axons at nodes of Ranvier. Nat Rev Neurosci 4:968-980.

Quintana A, Schwarz EC, Schwindling C, Lipp P, Kaestner L, Hoth M (2006) Sustained activity of calcium release-activated calcium channels requires translocation of mitochondria to the plasma membrane. J Biol Chem 281:40302-40309.

Saotome M, Safiulina D, Szabadkai G, Das S, Fransson A, Aspenstrom P, Rizzuto R, Hajnoczky G (2008) Bidirectional Ca2+-dependent control of mitochondrial dynamics by the Miro GTPase. Proc Natl Acad Sci U S A 105:20728-20733.

Smith KJ, McDonald WI (1980) Spontaneous and mechanically evoked activity due to central demyelinating lesion. Nature 286:154-155.

Smith KJ, McDonald WI (1999) The pathophysiology of multiple sclerosis: the mechanisms underlying the production of symptoms and the natural history of the disease. Philos Trans R Soc Lond B Biol Sci 354:1649-1673.

Stys PK (2005) General mechanisms of axonal damage and its prevention. J Neurol Sci 233:3-13.

Sun BB, Chiu SY (1999) N-type calcium channels and their regulation by $\mathrm{GABA}_{\mathrm{B}}$ receptors in axons of neonatal rat optic nerve. J Neurosci 19:5185-5194.

Thastrup O, Cullen PJ, Drobak BK, Hanley MR, Dawson AP (1990) Thapsigargin, a tumor promoter, discharges intracellular $\mathrm{Ca} 2+$ stores by spe- 
cific inhibition of the endoplasmic reticulum Ca2(+)-ATPase. Proc Natl Acad Sci U S A 87:2466-2470.

Verbny Y, Zhang CL, Chiu SY (2002) Coupling of calcium homeostasis to axonal sodium in axons of mouse optic nerve. J Neurophysiol 88:802816.

Wang X, Schwarz TL (2009) The mechanism of Ca2+-dependent regulation of kinesin-mediated mitochondrial motility. Cell 136:163174.

Waxman SG (2006) Axonal conduction and injury in multiple sclerosis: the role of sodium channels. Nat Rev Neurosci 7:932-941.

Waxman SG (2008) Axonal dysfunction in chronic multiple sclerosis: meltdown in the membrane. Ann Neurol 63:411-413.

Yang F, He XP, Russell J, Lu B (2003) Ca2 + influx-independent synaptic potentiation mediated by mitochondrial $\mathrm{Na}(+)-\mathrm{Ca} 2+$ exchanger and protein kinase C. J Cell Biol 163:511-523.

Yi M, Weaver D, Hajnoczky G (2004) Control of mitochondrial motility and distribution by the calcium signal: a homeostatic circuit. J Cell Biol 167:661-672.

Young EA, Fowler CD, Kidd GJ, Chang A, Rudick R, Fisher E, Trapp BD (2008) Imaging correlates of decreased axonal $\mathrm{Na}+/ \mathrm{K}+$ ATPase in chronic multiple sclerosis lesions. Ann Neurol 63:428-435.

Zhang CL, Verbny Y, Malek SA, Stys PK, Chiu SY (2004) Nicotinic acetylcholine receptors in mouse and rat optic nerves. J Neurophysiol 91:1025-1035.

Zhang CL, Wilson JA, Williams J, Chiu SY (2006) Action potentials induce uniform calcium influx in mammalian myelinated optic nerves. J Neurophysiol 96:695-709. 